\title{
Physical Observable for One-Dimensional Competing Many-Body Interactions
}

\author{
Chunhuan Xiang1, Honglei Wang2* (1) \\ ${ }^{1}$ School of Public Health and Management, Chongqing Medical University, Chongqing, China \\ ${ }^{2}$ College of Medical Informatics, Chongqing Medical University, Chongqing, China \\ Email: *w8259300@163.com
}

How to cite this paper: Xiang, C.H. and Wang, H.L. (2019) Physical Observable for One-Dimensional Competing Many-Body Interactions. Open Journal of Applied Sciences, 9, 595-600.

https://doi.org/10.4236/ojapps.2019.97047

Received: June 20, 2019

Accepted: July 22, 2019

Published: July 25, 2019

Copyright $\odot 2019$ by author(s) and Scientific Research Publishing Inc. This work is licensed under the Creative Commons Attribution International License (CC BY 4.0).

http://creativecommons.org/licenses/by/4.0/

\begin{abstract}
The one-dimensional 3-spin couplings and 2-spin competing many-body interactions with magnetic fields are an interesting field in quantum system, which is theoretically analysed in an experimental configuration. In this paper, we study the physical observable information for one-dimensional competing many-body interactions. The figure for symmetry broken state density, local order parameter, scaling of entanglement entropy is shown. The results agree with the phase diagram.
\end{abstract}

\section{Keywords}

Local Order Parameter, Phase Diagram, Entanglement Entropy, Scaling

\section{Introduction}

Quantum phase transition occurs as a result of a sudden change in the ground state as a system's parameter (such as external field) is slowly changed [1] [2] [3] [4] [5]. Quantum fluctuations, instead of thermal fluctuations, drive such transitions in absolute zero temperature. On the other hand, experiments in quantum simulators, such as ultra-cold atoms in optical lattices and trapped ions can access the dynamical properties of closed quantum many-body systems far from equilibrium [6] [7] [8] [9]. The one-dimensional competing many-body interactions are an important model and had been researched in experiment, however, trapped ions can be used to study exotic quantum phases which do not have a counterpart in nature.

The numerical simulation algorithm is made much progress in time and space, which is used to understand the collective behavior of quantum many-body systems. More is different. The collective behavior is a formidable challenge due to the exponential growth of Hilbert space dimension with system size. N. G. Vidal 
and his colleagues had introduced the matrix product states [10] [11] [12] [13] [14] in one spatial dimension and the projected entanglement-pair states [15] [16]

[17] in two and higher spatial dimensions. The good approximation ground-state wave-function for transitionally invariant quantum systems on an infinite-size lattice can be obtained by the variational algorithm.

In this paper, the figure for symmetry broken state density, local order parameter, scaling of entanglement entropy and ground state fidelity per latter site is obtained by the approximation ground-state wave-function.

\section{The Hamiltonian and Ground-State Phase Diagram}

The Hamiltonian for the one-dimensional competing many-body interactions [18] is given as the follow

$$
H=J_{2} \sum_{j} \sigma_{j}^{z} \sigma_{j+1}^{z}+J_{3} \sum_{j} \sigma_{j}^{z} \sigma_{j+1}^{z} \sigma_{j+2}^{z}-h \sum_{j} \sigma_{j}^{x} .
$$

where $J_{2}$ and $J_{3}$ are the exchange couplings for two-spins and three-spins, respectively, $h$ is the external magnetic field, and $\sigma$ is the spin-1/2 operator on the site $j$.

$$
\sigma_{x}=\left(\begin{array}{ll}
0 & 1 \\
1 & 0
\end{array}\right), \quad \sigma_{z}=\left(\begin{array}{cc}
1 & 0 \\
0 & -1
\end{array}\right)
$$

The competition between the different terms of Hamiltonian determined the ground state. With the exchange couplings $J_{2}=0$ or $J_{3}=0$, Equation (1) is deduced into one-dimensional quantum Ising model. When exchange coupling $J_{2}(>0)$ induces antiferromagnetic $(\mathrm{AF})$ order, $J_{3}(<0)$ will be shown to induce a novel ferrimagnetic (F) phase, and $h(>0)$ encourages the system to lie in a disordered paramagnetic $(\mathrm{P})$ regime, the phase diagram is shown in Figure 1. In this paper, we set $h=1$ and $J_{2}=0$, that is to say, we study the line along the $x$-direction. The 3 -spin interactions induce a novel quantum phase, which is no longer exactly solvable, but shows self-duality properties [19]. The value $\left|J_{3}\right| / h=1$ means a four-fold ferrimagnetic state emerged, which is improved by the symmetry broken state density and shown in Figure 2.

The symmetry broken state density of the Hamiltonian (1) with $h=1, J_{2}=0$ and $J_{3}=2.8000$ approximation ground state wave-functions are obtained with the truncation dimension $\chi=4$. We pick up 80 wavefunctions and label one state from the four-fold ferrimagnetic state. The times we pick up are 100,000. The line for green-ball is yield. The same method is for 120 wavefunctions with the triangle line. From Figure 2, we know that the chance for the 20 labeled states of the 80 wavefunctions arrives to the peak, the maximum appears for the 30 labeled states of the 120 wavefunctions.

\section{Physical Observable for the Competing Many-Body Interactions}

The local order parameter is an important observable in phase transition field, which is obtained by order parameter and the good approximation ground state wavefunction. The simulation results of the local order parameter for Equation 


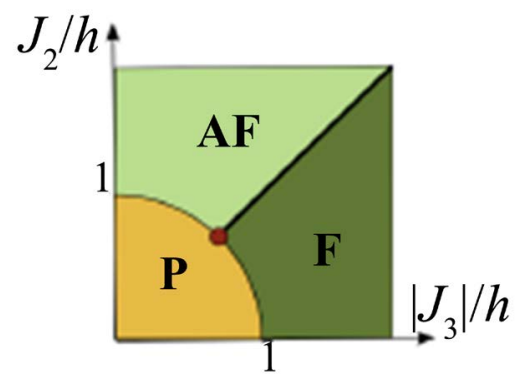

Figure 1. Ground state phase diagram [18] of the Hamiltonian (1). The phase AF, F and $\mathrm{P}$ are separated with the solid line in different color. The red point is the tri-critical point among the AF, F and $\mathrm{P}$ phase.

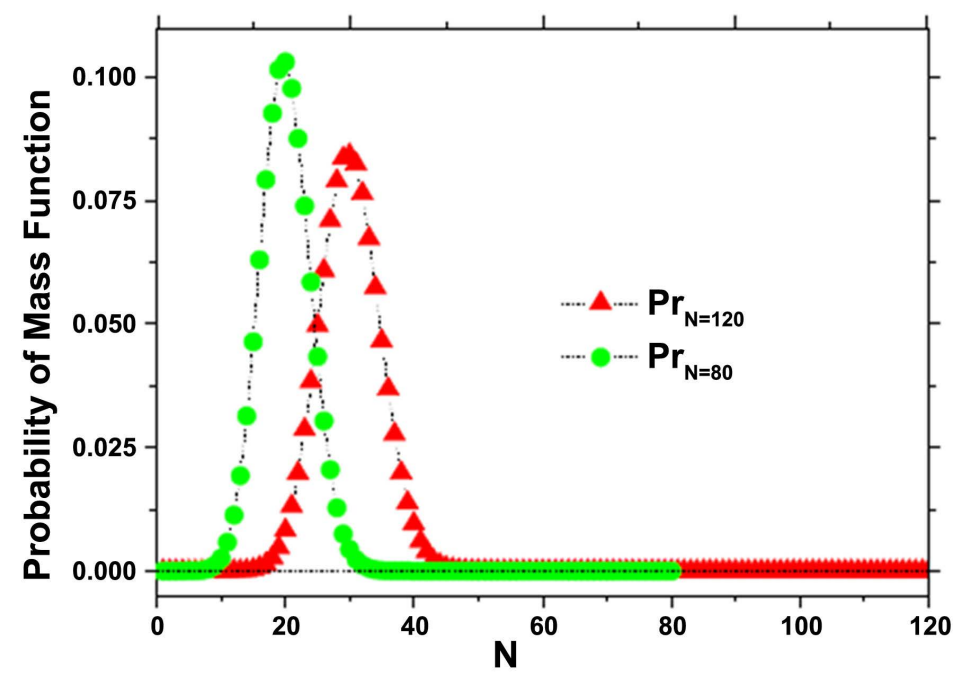

Figure 2. The symmetry broken state density of the Hamiltonian (1) with $h=1, J_{2}=0$ and $J_{3}=2.8000$ approximation ground state wave-functions are obtained with the truncation dimension $\chi=4$. We pick up 80 wavefunctions and label one state from the four-fold ferrimagnetic state. The times we pick up are 100,000. The line for green-ball is yield. The same method is for 120 wavefunctions with the triangle line.

(1) are shown in Figure 3 with truncation dimension $\chi=4,8,16,32$ in different label, respectively. With the truncation dimension is larger and larger, the phase transition point is moving to the point $J_{3}=1$, which is the phase transition point in theory. The inset figure is the extrapolation for the phase transition point given by the truncation dimension $\chi=4,8,16,32$, the result is well agree with the $J_{3}=1$.

The amount of entanglement can be quantified in terms of the von Neumann entropy, which is known to obey scaling properties for an infinite chain. We may try to find the exact amount of entanglement which is captured in different truncation dimension by using matrix product states. The maximum entanglement entropy for each truncation dimension meets the scaling relationship

$$
S=a \log (\chi)+b
$$

The results are $a=0.225, b=-0.898$ with the truncation dimension $\chi=8,16$, $20,24,28,32,48$. The scaling relationship for (1) is shown in Figure 4. The ground state fidelity is shown in Figure 5. 


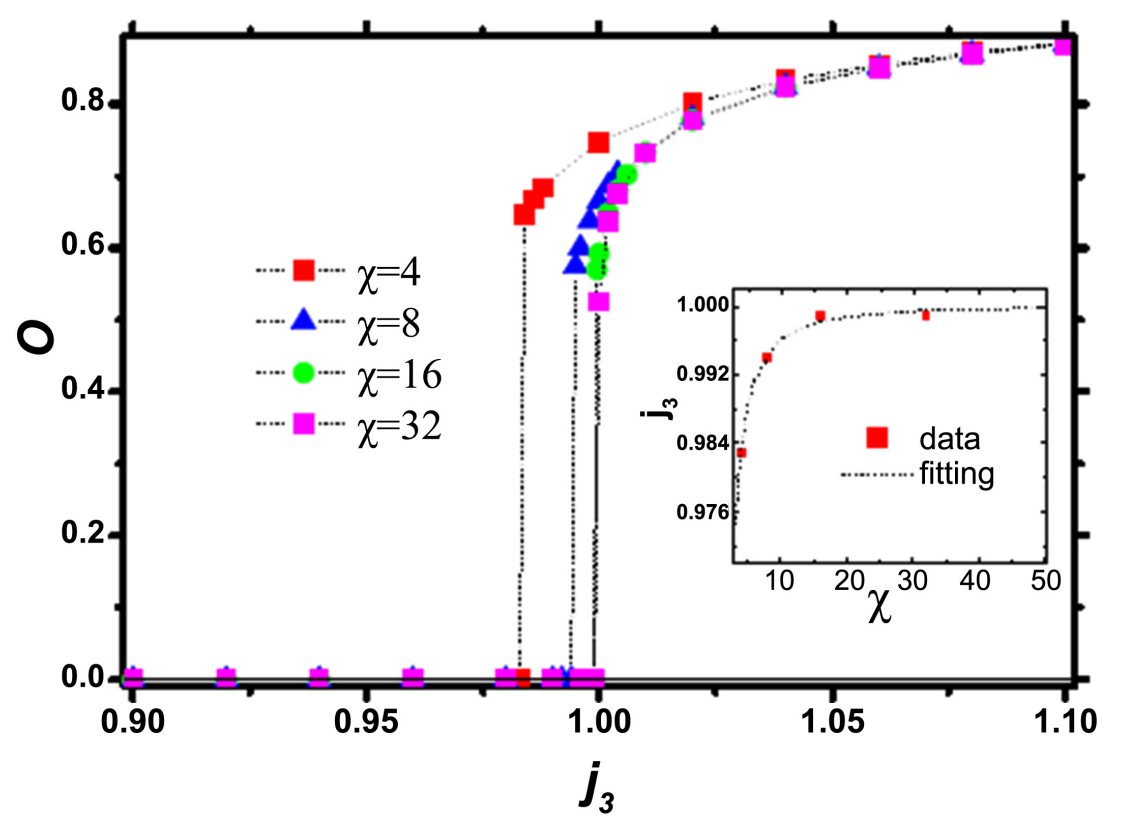

Figure 3. The local order parameter for the Hamiltonian (1) with $h=1, J_{2}=0$ and $J_{3}$ as the control parameter, the truncation dimensions are shown with $\chi=4,8,16,32$. The inset figure is the extrapolation for the phase transition point given by the truncation dimension $\chi=4,8,16,32$. The solid lines are the guide for eye.

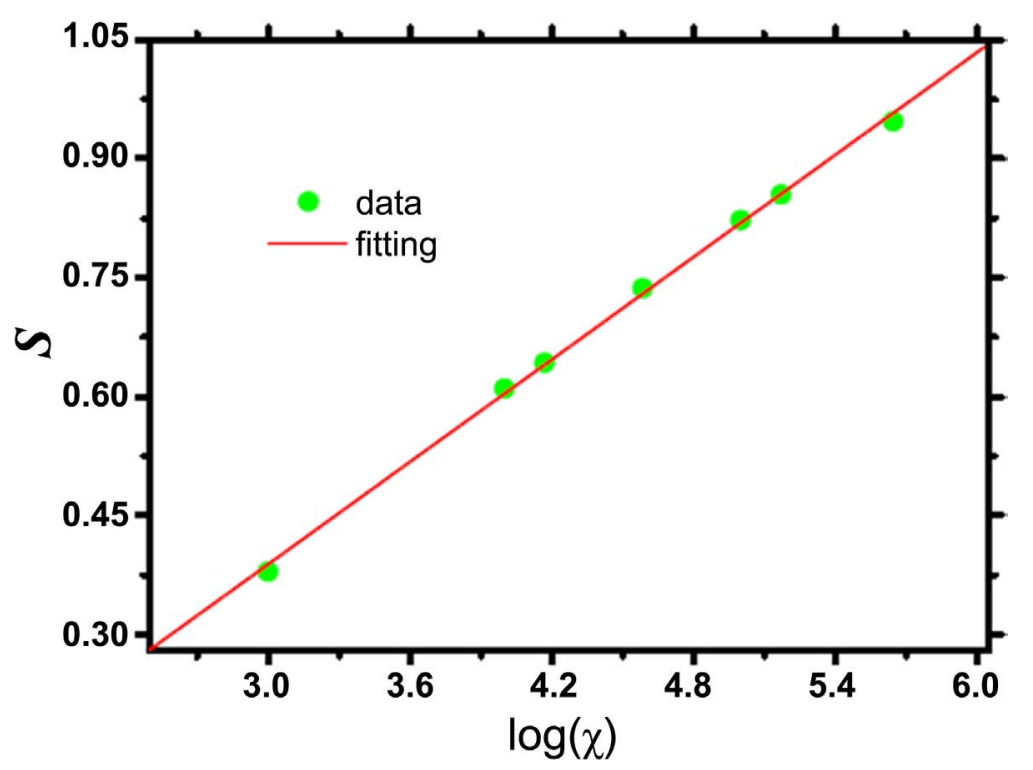

Figure 4. The scaling relationship for entanglement entropy of the Hamiltonian (1) with truncation dimension $\chi=8,16,20,24,28,32,48$, the parameter for the Equation (2) is a $=0.225, b=-0.898$. The solid lines are the guide for eye.

\section{Summary}

The one-dimensional competing many-body interactions are investigated by using matrix product states. Although the amount of entanglement supported by the matrix product states approximation is limited by the size $\chi$ of the matrices, we obtained interesting results. The physical observable parameters: local order 


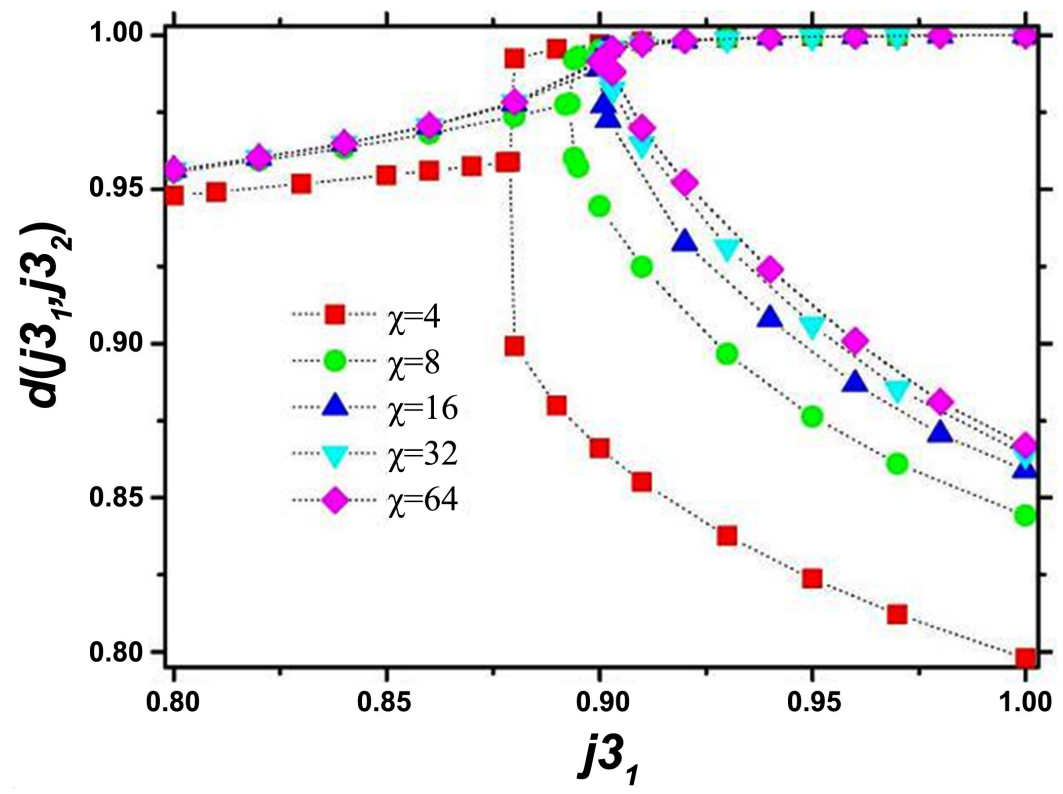

Figure 5. The ground state fidelity of the Hamiltonian (1) with truncation dimension $\chi=$ $4,8,16,32,64$. The bifurcation point given by the fidelity is same as the point given by local order parameter with the corresponding dimension $\chi$. The dot lines are the guide for eye.

parameter and scaling relationship of the entanglement entropy are shown in this paper. Besides, we also give the symmetry broken state density. All the results well agree with the known phase diagram. The numerical work we have performed is qualitatively valid for other models.

\section{Conflicts of Interest}

The authors declare no conflicts of interest regarding the publication of this paper.

\section{References}

[1] Sachdev, S. (2013) Quantum Phase Transitions. Cambridge Univ. Press, Cambridge.

[2] Langen, T., Geiger, R., Kuhnert, M., Rauer, B. and Schmiedmayera, J. (2013) Dynamical Phase Transition in the 1D-Transverse Field Ising Chain Characterized by the Transverse Magnetization Spectral Function. Nature Physics, 9, 640. https://doi.org/10.1038/nphys2739

[3] Langen, T., Erne, S., Geiger, R., Rauer, B., Schweigler, T., Kuhnert, M., Rohringer, W., Mazets, I., Gasenzer, T. and Schmiedmayer, J. (2015) The Principle of Antagonism Ensures Protein Targeting Specificity at the Endoplasmic Reticulum. Science, 348, 201-207. https://doi.org/10.1126/science.1257026

[4] Polkovnikov, A., Sengupta, K., Silva, A. and Vengalattore, M. (2011) Colloquium: Nonequilibrium Dynamics of Closed Interacting Quantum Systems. Reviews of Modern Physics, 83, 863-883. https://doi.org/10.1103/RevModPhys.83.863

[5] Gogolin, C. and Eisert, J. (2016) Equilibration, Thermalisation, and the Emergence of Statistical Mechanics in Closed Quantum Systems. Reports on Progress in Physics, 79, Article ID: 056001. https://doi.org/10.1088/0034-4885/79/5/056001 
[6] Bloch, I., Dalibard, J. and Zwerger, W. (2008) Many-Body Physics with Ultracold Gases. Reviews of Modern Physics, 80, 885-964. https://doi.org/10.1103/RevModPhys.80.885

[7] Blatt, R. and Roos, C.F. (2012) Quantum Simulations with Trapped Ions. Nature Physics, 8, 277-284. https://doi.org/10.1038/nphys2252

[8] Bloch, I., Dalibard, J. and Nascimb'ene, S. (2012) Quantum Simulations with Ultracold Quantum Gases. Nature Physics, 8, 267-276. https://doi.org/10.1038/nphys2259

[9] Georgescu, I.M., Ashhab, S. and Nori, F. (2014) Quantum Simulation. Reviews of Modern Physics, 86, 153. https://doi.org/10.1103/RevModPhys.86.153

[10] Fannes, M., Nachtergaele, B. and Werner, R.F. (1992) Finitely Correlated States on Quantum Spin Chains. Communications in Mathematical Physics, 144, 443-490. https://doi.org/10.1007/BF02099178

[11] Ostlund, S. and Rommer, S. (1995) Thermodynamic Limit of Density Matrix Renormalization. Physical Review Letters, 75, 3537-3540. https://doi.org/10.1103/PhysRevLett.75.3537

[12] Perez-Garcia, D., Verstraete, F., Wolf, M.M. and Cirac, J.I. (2007) Matrix Product State Representations. Quantum Information and Computation, 7, 401-430.

[13] Verstraete, F., Porras, D. and Cirac, J.I. (2004) Density Matrix Renormalization Group and Periodic Boundary Conditions: A Quantum Information Perspective. Physical Review Letters, 93, Article ID: 227205. https://doi.org/10.1103/PhysRevLett.93.227205

[14] Vidal, G. (2007) Classical Simulation of Infinite-Size Quantum Lattice Systems in One Spatial Dimension. Physical Review Letters, 98, Article ID: 070201. https://doi.org/10.1103/PhysRevLett.98.070201

[15] Takasaki, H., Hikihara, T. and Nishino, T. (1999) Fixed Point of the Finite System DMRG. Journal of the Physical Society of Japan, 68, 1537-1540. https://doi.org/10.1143/JPSJ.68.1537

[16] Nishino, T., Okunishi, K., Hieida, Y., Maeshima, N. and Akutsu, Y. (2000) Self-Consistent Tensor Product Variational Approximation for 3D Classical Models. Nuclear Physics B, 575, 504. https://doi.org/10.1016/S0550-3213(00)00133-4

[17] Verstraete, F. and Cirac, J.I. (2004) Renormalization Algorithms for Quantum-Many Body Systems in Two and Higher Dimensions.

[18] Bermudez, A., Porras, D. and Martin-Delgado, M.A. (2009) Competing Many-Body Interactions in Systems of Trapped Ions.

[19] Penson, K.A., Jullien, R. and Pfeuty, P. (1982) Phase Transitions in Systems with Multispin Interactions. Physical Review B, 26, 6334. https://doi.org/10.1103/PhysRevB.26.6334 